\title{
Non-invasive vagus nerve stimulation normalizes food liking and improves liking ratings in depression
}

Vincent Koepp ${ }^{1-2^{*}}$, Johannes Klaus ${ }^{1 *}$, Magdalena Ferstl ${ }^{1}$, Franziska K. Müller ${ }^{1}$, Anne Kühnel ${ }^{1,3}$, \& Nils B. Kroemer ${ }^{1, c}$

${ }^{1}$ Department of Psychiatry and Psychotherapy, Tübingen Center for Mental Health, University of Tübingen, Tübingen, Germany

${ }^{2}$ Institute for Psychology, Otto-von-Guericke University Magdeburg, Magdeburg, Germany

3 Department of Translational Research in Psychiatry, Max Planck Institute of Psychiatry and International Max Planck Research School for Translational Psychiatry (IMPRS-TP), Munich, Germany

*equal contribution

Corresponding author ${ }^{\mathrm{c}}$

Dr. Nils B. Kroemer, nils.kroemer@uni-tuebingen.de

Calwerstr. 14, 72076 Tübingen, Germany 


\section{Abstract}

Introduction: The vagus nerve plays a prominent role in the regulation of food reward and energy metabolism. However, previous studies using vagus nerve stimulation yielded conflicting results regarding changes in food reward in healthy participants and participants with major depressive disorder (MDD), for which vagal nerve stimulation is an effective treatment.

Methods: We investigated the acute effects of right transcutaneous auricular vagus nerve stimulation (taVNS) on ratings of liking and wanting of food and non-food items in 63 participants, including 31 patients with MDD. To test for taVNS-induced changes and interactions with between-subject variables group (MDD vs. healthy controls) and questionnaire scores as well as within-subject variables, we performed linear mixed-effects analysis. In addition, we assessed whether individual taVNS-induced changes in food reward ratings were dependent on average ratings.

Results: taVNS increased liking of food cues in participants with MDD ( $p=0.023)$, but not in healthy participants $(p=0.657)$. Specifically, taVNS induced larger improvements in liking ratings with increasing scores of anhedonia $(p=0.029)$. Notably, across all participants, taVNS reduced the variance of food liking compared to sham, suggesting that taVNS normalizes extreme liking ratings towards moderate levels $(p=0.039)$.

Discussion: Our results show that taVNS acutely ameliorates hedonic responses in MDD suggesting that it could provide an effective treatment of anhedonia. Since anhedonia is difficult to treat with conventional therapies, taVNS may provide a powerful adjuvant to rapidly improve motivational deficiencies. 


\section{Introduction}

Major depressive disorder (MDD) is a highly prevalent, disabling, and recurrent disorder (Whiteford et al., 2013). One in five adults is affected over the course of their life and the social and economic burden is substantial (Hasin et al., 2018). Despite this prevalence, exact neurobiological pathomechanisms are still poorly understood. Since MDD is a heterogeneous syndrome rather than a unitary disorder (Pizzagalli, 2014; Vrieze et al., 2014), there is an increasing focus on specific symptoms to unravel the neurobiological mechanisms of MDD and improve treatment specificity (Keedwell et al., 2005; Keller et al., 2013; Young et al., 2016). One of the core symptoms of MDD is anhedonia, the experienced loss of pleasure, motivation, and interest to pursue rewards including food. It affects up to $70 \%$ of MDD patients (Shankman et al., 2014) and is seen as a predictor of suicide and poorer response to pharmacological treatments (Craske et al., 2016). Crucially, deficits in experiencing pleasure can arise from impaired reward processing (Rizvi et al., 2016). The reward process entails appetitive and consummatory phases, which are dominated by either "wanting" (i.e., incentive salience to obtain a reward) or "liking" (i.e., reaction to hedonic impact) (Rømer Thomsen et al., 2015). Food is a primary reward and decreased pleasure in anticipating or consuming palatable food is typically an indication of anhedonia (Coccurello, 2019). Thus, by investigating individual reactivity to food cues in patients with MDD combined with targeted neuromodulation, we can identify potential ways to improve reward function.

Invasive vagus nerve stimulation (iVNS) is an approved and effective therapeutic tool for treatment-resistant MDD (Bottomley et al., 2020; Wu et al., 2018). Due to its bidirectional communication with the gastrointestinal tract, the vagus nerve forms an essential part of the gut-brain axis (Breit et al., 2018) and plays a pivotal role in the regulation of food intake and metabolic processes according to homeostatic needs (Berthoud, 2008; de Araujo et al., 2012; de Lartigue \& Diepenbroek, 2016; Waise et al., 2018; Yuan \& Silberstein, 2016). It contributes to energy homeostasis by conveying satiety (Chaudhri et al., 2008; de Lartigue \& Diepenbroek, 2016; Peters et al., 2006; Waise et al., 2018) and motivational signals (Han et al., 2018; Neuser et al., 2020). Mimicking vagal satiety signaling by applying iVNS has been shown to alter food intake and induce weight loss in animal studies (Dai et al., 2020; Sobocki et al., 2005; Val-Laillet et al., 2010), although results from human studies remain inconsistent to date (Pelot \& Grill, 2018). Notably, vagal afferent signals have been shown to modulate mesolimbic dopamine signaling, which is associated with motivation and reward processing (de Araujo et al., 2012; Han et al., 2018) indicating that VNS might also affect motivational salience ('wanting') or hedonic value ('liking') of food stimuli. Relatedly, we have shown that non-invasive transcutaneous auricular VNS (taVNS) altered reward learning and boosted the vigor to work for rewards (Neuser et al., 2020; Kühnel et al., 2020), which is in line with altered 
monoaminergic neurotransmission. However, three recent studies showed no evidence of taVNS-induced effects on liking and wanting ratings of food or on the amount of ingested food in healthy participants (Alicart et al., 2020; F.K. Müller et al., 2021; Obst et al., 2020). In contrast, in a small pilot study, Öztürk et al. (2020) found taVNS-induced increases in food liking after the consumption of low-fat food. Furthermore, in a sample of participants with MDD, Bodenlos et al. (2007) reported a significant increase of individual differences in cravings of sweet food. In conclusion, VNS may affect food-reward responses with preliminary evidence indicating that stimulation effects could differ between healthy control participants (HCP) and people suffering from MDD and anhedonia.

To compare the effects of non-invasive taVNS on ratings of food reward in participants with MDD versus HCP, we used a food-cue reactivity task (FCR; F.K. Müller et al., 2021) while administering right-sided taVNS or sham stimulation (Ellrich, 2011; Peuker \& Filler, 2002). Using a randomized cross-over design, we investigated if taVNS alters subjective liking and wanting of food cues and whether taVNS-induced effects differ in participants with MDD. To account for interindividual heterogeneity of MDD symptoms, we examined whether taVNSinduced changes are associated with anhedonia (measured with the SHAPS questionnaire) and reduced reward approach (measured with the behavioral activation system, BAS, questionnaire). We found that taVNS increased food liking in participants with MDD and gains were correlated with anhedonia, exerting a normalizing effect on food liking in all participants.

\section{Methods}

\section{Participants}

For the current study, we enrolled $34 \mathrm{HCP}$ and 31 participants with MDD matched on the group level for age, sex and BMI. Participants were between 19 and 61 years old $(\mathrm{M}=$ 34.1), right-handed, German speaking, physically healthy and did not have other mental disorders apart from anxiety or trauma-related disorders in the MDD and anxiety disorders without depressive symptoms $(\mathrm{BDI}<7)$ in the HCP group. HCP were only included if they never fulfilled the criteria for a major depressive episode. For the reported analyses, we excluded two HCPs who did not complete the second session (total $\mathrm{N}=63$, MDD=31). MDD diagnosis was based on telephone screenings, where we interviewed candidates according to the German SCID-II (Fydrich et al., 1997). From the 31 participants with MDD, 20 were taking antidepressant medication at the time of the telephone screening (Fig. S1-2). In each session, participants received snacks and money they had won during the behavioral session. 
After completion of the second session, participants received a fixed compensation of $32 €$ or an equivalent partial course credit. The study was approved by the local ethics committee (the Institutional Review Board of the Faculty of Medicine, University of Tübingen) and was conducted in accordance with the ethical code of the World Medical Association (Declaration of Helsinki).

\section{Experimental procedure}

The study followed a single-blinded, randomized cross-over design. Similar to a previous study (F.K. Müller et al., 2021), each participant completed two sessions receiving either sham or taVNS with a delay of 2 to $15 \mathrm{~d}$ between sessions (median $=4 \mathrm{~d}$ ). Both sessions started at approximately the same time and participants were asked to fast (including caffeinated drinks) for 3-5 h ( $M=3.9$; corresponding to a state of neither hungry, nor full, Fig. S3) and refrain from consuming alcohol $48 \mathrm{~h}$ prior to each session. This was different compared to (F.K. Müller et al., 2021) where sessions were scheduled in the morning after an overnight fast. After each session, participants further guessed the stimulation condition (sham or taVNS). Correct responses occurred at chance level (recorded guesses: 126, correct guesses: 69 , accuracy: $54.8 \%, p_{\text {binom }}=0.33$ ), indicating that blinding was successful.

Each session started with measurements of weight, height, hip and waist circumference as well as pulse rate. Next, participants completed the German version of the Beck Depression Inventory II (BDI-II) (Beck et al. 1996; Hautzinger et al. 2006) and the first of the three mood assessments collected throughout the session (Ferstl et al., 2021). The mood state was assessed with the German Positive and Negative Affect Schedule (PANAS) (Krohne et al., 1996; Watson et al., 1988) which was presented as visual analogue scale (VAS) (Crichton, 2001). Next, the taVNS electrode was applied and stimulation strength was calibrated. The first experimental task was the FCR task which started 5 to 10 min after stimulation onset and lasted approximately $30 \mathrm{~min}$. The FCR task was then followed by an effort allocation task (Neuser et al., 2020) and a reward learning task (Kühnel et al., 2020). After the completion of the tasks, the BDI-Il questionnaire was administered for a second time alongside other questionnaires (not reported here). Each of the two sessions took about $2.5 \mathrm{~h}$. 
Table 1: Demographics and Questionnaire Scores.

\begin{tabular}{|c|c|c|c|c|c|}
\hline Variable & $\mathbf{N}$ & $\begin{array}{l}\text { Overall, } \\
N=63^{1}\end{array}$ & $\begin{array}{c}\text { HCP, } \\
N=32^{1}\end{array}$ & $\begin{array}{l}\text { MDD, } \\
\mathrm{N}=31^{1}\end{array}$ & $p$-value \\
\hline Sex & 63 & & & & - \\
\hline Male & & $20(32 \%)$ & $11(34 \%)$ & $9(29 \%)$ & - \\
\hline Female & & $43(68 \%)$ & $21(66 \%)$ & $22(71 \%)$ & - \\
\hline Age & 63 & $34.1(13.2)$ & $36.5(14)$ & $31.6(12.1)$ & 0.146 \\
\hline $\begin{array}{l}\text { Body Mass } \\
\text { Index (BMI) }\end{array}$ & 63 & $24.3(3.4)$ & $24.1(3.3)$ & $24.5(3.5)$ & 0.595 \\
\hline $\begin{array}{l}\text { Waist-to-Hip } \\
\text { Ratio (WtHR) }\end{array}$ & 63 & $0.8(0.1)$ & $0.8(0.1)$ & $0.8(0.1)$ & 0.991 \\
\hline $\begin{array}{l}\text { Beck } \\
\text { Depression } \\
\text { Inventory (BDI- } \\
\text { II) }\end{array}$ & 63 & $12.4(10.6)$ & $4.3(4.5)$ & 20.8 (8.3) & $<0.001$ \\
\hline $\begin{array}{l}\text { Snaith Hamilton } \\
\text { Pleasure Scale } \\
\text { (SHAPS) }\end{array}$ & 63 & $2.4(2.9)$ & $1.0(2.5)$ & $3.9(2.5)$ & $<0.001$ \\
\hline $\begin{array}{l}\text { Behavioral } \\
\text { Activation } \\
\text { System (BAS) }\end{array}$ & 63 & $37.9(5.8)$ & $39.9(4.6)$ & 35.7 (6.3) & 0.004 \\
\hline
\end{tabular}

1n (\%); Mean (SD)

Note. $P$-values are results of a Student's two-sample $t$-test.

\section{Right transcutaneous vagus nerve stimulation}

Transcutaneous vagus nerve stimulation of the auricular branch followed the same protocol as described in our previous work (F.K. Müller et al., 2021; Neuser et al., 2020). We used the Cerbomed NEMOS (Cerbomed GmbH, Erlangen) device which delivers $25 \mathrm{~Hz}$ stimulation in a biphasic manner, alternating between $30 \mathrm{~s}$ stimulation and $30 \mathrm{~s}$ pause. Depending on the stimulation condition, electrodes were placed either at the cymba conchae of the right external ear for taVNS or at the right earlobe, which is not innervated by the vagus nerve, for sham stimulation (Frangos et al., 2015; Peuker \& Filler, 2002) (Fig. 1). The stimulation protocol followed the recommendations in (Farmer et al., 2020). To account for 
individual differences in the subjective experience of the stimulation, strength was individually adjusted using pain VAS. Strength was increased until participants reported a 'tingling' sensation below the pain threshold on the VAS (Frangos et al., 2015) (stimulation: $M=1.24$ $\mathrm{mA}, S D=0.51$. sham: $M=1.41 \mathrm{~mA}, S D=0.54)$.

\section{Food-cue reactivity task}

Food wanting and liking ratings were measured using a FCR task (Fig. 1) that we have previously used to study taVNS effects in healthy participants (F.K. Müller et al., 2021). Briefly, participants rated pictures of food and non-food control items (stationery items) on separate liking and wanting VAS. The vertically labelled liking scale ranged from -100 (strongest disliking imaginable) to 100 (strongest liking imaginable) (Lim et al., 2009) while the wanting scale was oriented horizontally and ranged from 0 (not wanted at all) to 100 (strongly wanted). In total, 60 food and 20 non-food images were selected from a set of 80 food and 40 non-food items (Charbonnier et al., 2016) and presented twice per session. Every participant was presented with an identical image sample, but the presentation order was randomized across subjects and sessions. Food images were further classified by caloric density (high-caloric vs. low-caloric food) and taste quality (sweet vs. savory) according to Charbonnier et al. (2016). Classification resulted in an almost even number of images per category. The images were shown for $1.5 \mathrm{~s}$ each and ratings on the VAS could be submitted within a $2 \mathrm{~s}$ interval by moving the left joystick of an Xbox 360 controller and confirming the choice with a button press (Fig. 1). 


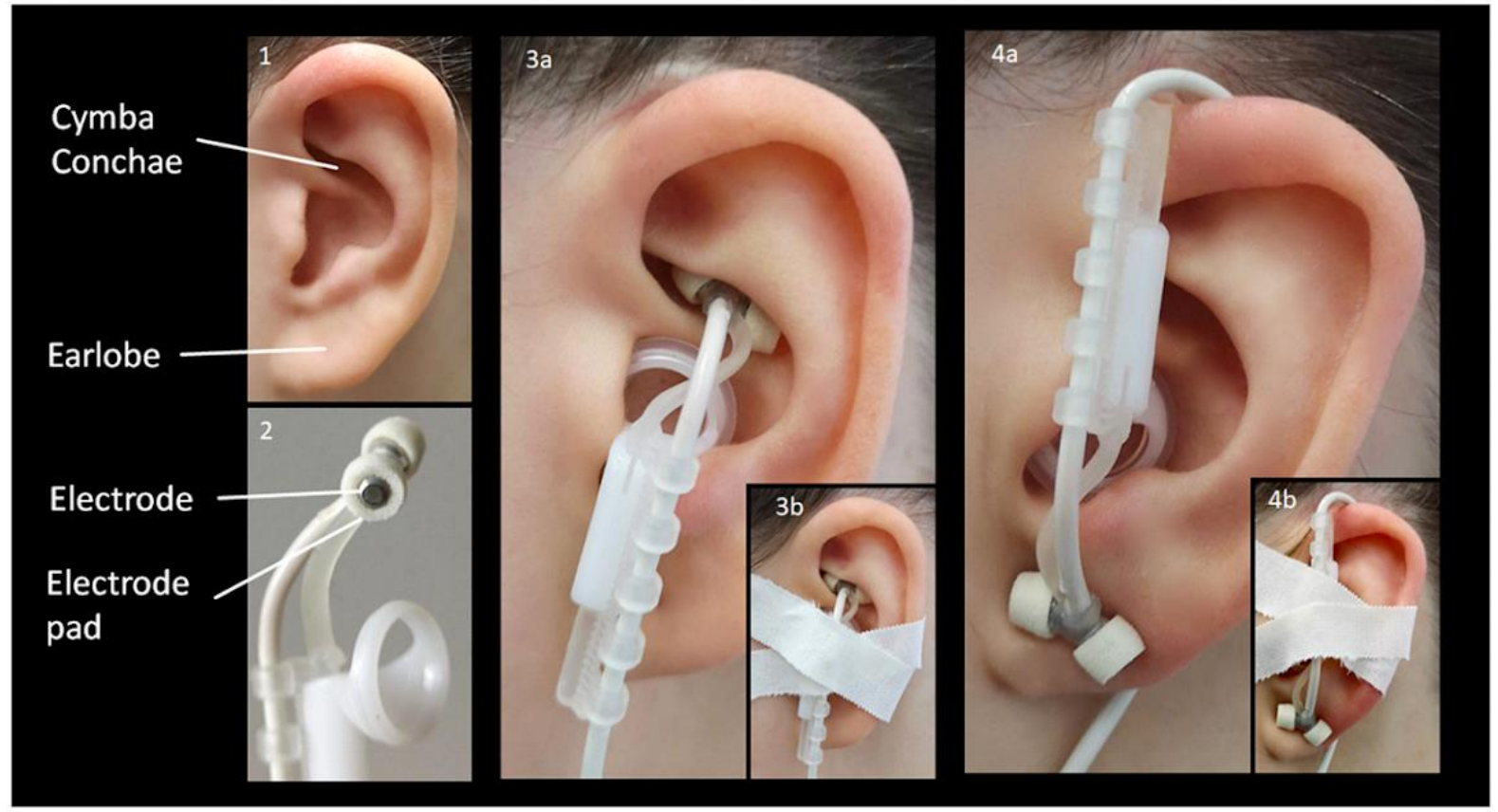

b

\section{Procedure}

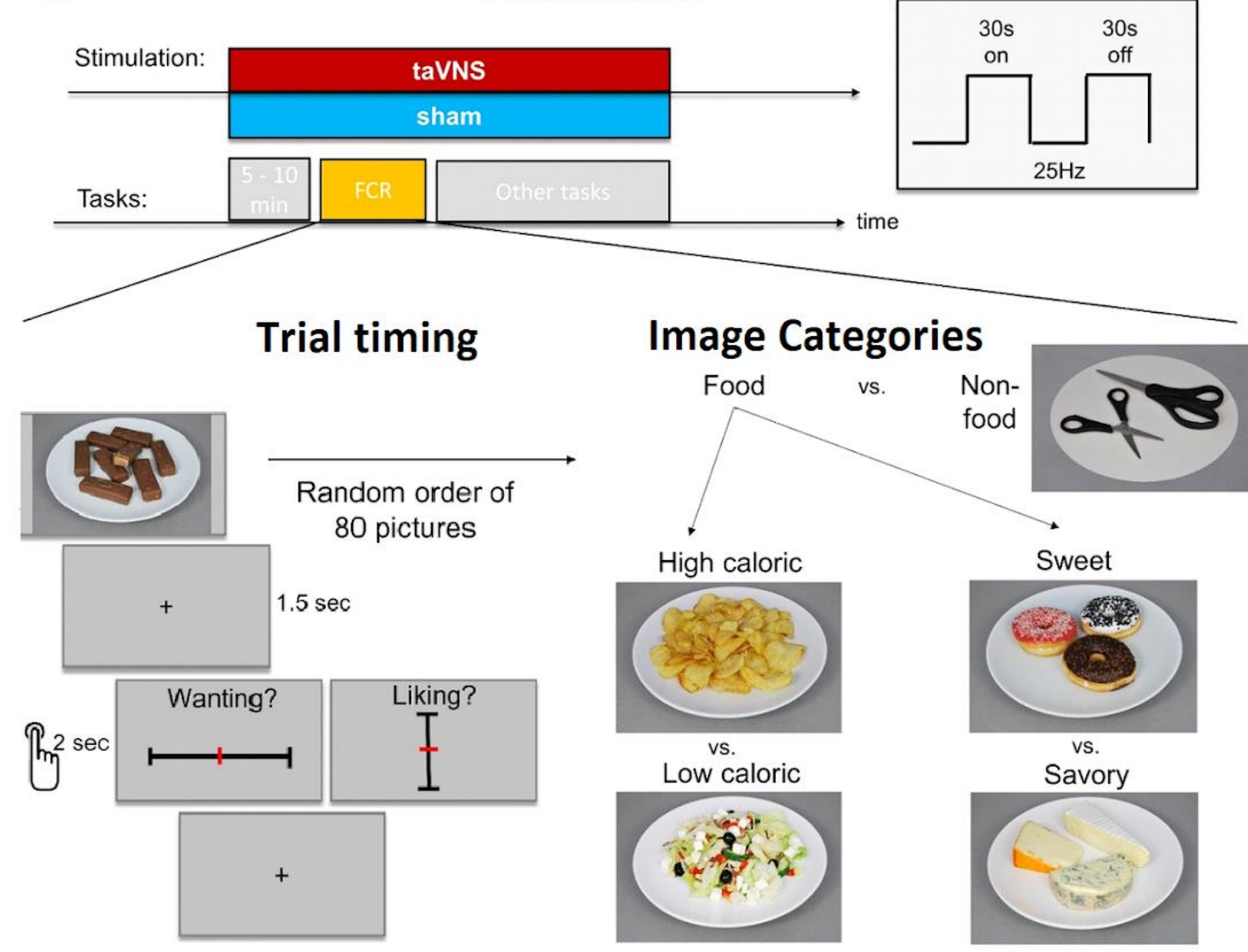

Figure 1. Schematic summary of electrode placement and experimental procedure (adapted from F.K. Müller et al. (2021)). A: The electrode is placed at the cymba conchae of the inner ear during taVNS (3a) and at the earlobe during sham (4a). In both cases the electrode is secured by tape. B: The biphasic on- and off-duty stimulation starts 5-10 minutes before the food-cue reactivity (FCR) task which is then followed by additional tasks. The FCR task required participants to submit their rating of liking and wanting of different food and non-food items. The food items can be further classified according to their caloric density (high-caloric vs. low-caloric food) and taste quality (sweet vs. savory). 


\section{Questionnaires}

Apart from the German BDI-II, participants received a link to fill out additional questionnaires at home including symptoms of altered reward processing. We used the German version of the Snaith Hamilton Pleasure Scale (SHAPS, (Franz et al., 2008; Snaith et al., 1995)) as a dimensional measure of anhedonia and the BAS subscale from the German version of the Behavioral Inhibition System/Behavioral Activation System questionnaire (BIS/BAS, (Carver \& White, 1994; Strobel et al., 2001)) as a continuous measure of the disposition to actively pursue rewards and subsequently tested associations between scores and taVNS effects.

\section{Data analysis}

To investigate taVNS effects and whether they differed in participants with MDD compared to HCP, we estimated a full mixed-effects model implemented in the 'ImerTest'package in R (Kuznetsova et al., 2017) using Satterthwaite's method for df calculation (we reported rounded df values in the results section). The main model (eq. 1) included the predictors Image Type (non-food vs. food items, centered), Stimulation (sham vs. taVNS, dummy coded) as well as their interaction as within-subject factors and Group (HCP vs. MDD, centered) as a participant-level factor allowing for cross-level interactions. We controlled for confounding effects of sex, age, and BMl by including them as additional participant-level factors (centered). To account for stimulation order effects (stimulation in first or second session), we entered Order (centered) and the Stimulation X Order interaction (see eq. 1).

Rating clmage_Type * cGroup * Stimulation + Stimulation * cOrder + cAge + cBMI + cSex + (1+ clmage_Type * Stimulation | ID)

The model included a random intercept as well as the random slopes for all withinsubject factors. To characterize potential differences in stimulation effects between HCP and MDD, we performed group-specific post hoc comparisons as implemented in the 'emmeans'package (Lenth, 2021) between taVNS and sham. For the post hoc comparisons and all analyses focusing on inter-individual differences, we included only food images. To reflect the complementary information provided by the ratings, we analyzed liking and wanting ratings in separate models.

Since stimulation effects or differences between patients with MDD and HCP could be specific for certain types of food (Bodenlos et al., 2007; Öztürk et al., 2020), we performed an additional analysis only including food images and partitioned them according to their caloric 
density (high-caloric vs. low-caloric food) and taste quality (sweet vs. savory). We adapted our main model and included Caloric Density, Taste, and their interactions instead of the Image Type factor as within-subject factors.

\section{Association of ratings during sham and questionnaire scores with stimulation effects}

To evaluate whether individual differences in reward processing are associated with taVNS-induced changes, we adapted our main model and included SHAPS and BAS scores (centered) and their interactions instead of the Group factor. Second, we investigated whether taVNS effects depend on individual rating intercepts (i.e., average ratings). To this end we tested differences in variance between stimulation conditions (sham vs. taVNS), by comparing a restrictive model with no correlations between the intercept and stimulation effects with a less restrictive model allowing for these correlations using a likelihood-ratio test (Westfall 2019).

\section{Statistical threshold and software}

All statistical tests were two-tailed with an alpha $\leq 0.05$ as significance threshold. For data analysis, we used the packages 'ImerTest' (Kuznetsova et al., 2017), 'emmeans' (Lenth, 2021). For data visualization, we used 'ggplot2' (Wickham, 2016) all within R Studio using and Rv4.1.1 (R Core Team, 2021).

\section{Results}

\section{taVNS increases food liking in participants with MDD}

To assess taVNS effects on liking and wanting ratings, we calculated mixed-effects models. As expected, both liking and wanting ratings were higher for food compared to nonfood pictures during sham (liking: $b=33.74, t(61)=10.43, p<0.001$; wanting: $b=28.56, t(61)=$ 10.34, $p<0.001$; Fig. 2). In contrast, liking and wanting did not differ between HCP and participants with MDD during sham (liking: $b=-2.18, t(58)=-0.71, p=0.483$; wanting: $b=1.06$, $t(59)=0.40, p=0.692$; negative value indicate lower values in patients with MDD) and there was no main effect of Stimulation (liking: $b=0.96, t(60)=1.01, p=0.316$; wanting: $b=0.59$, $t(60)=0.61, p=0.547)$. However, taVNS increased liking ratings more strongly in patients with MDD compared to HCP (Group X Stimulation. $b=4.63, t(60)=2.43, p=0.018$ ) while wanting ratings were not significantly affected (Group X Stimulation. $b=1.66, t(60)=0.86, p=0.396$; Fig. 2). 
To investigate differential stimulation effects between MDD and HCP groups on food images, we conducted group-specific post hoc comparisons (see Supplementary Information). taVNS significantly increased food liking in $\operatorname{MDD}(t(60)=2.33, p=0.023)$, but not in $\mathrm{HCP}(t(60)=$ $-0.45, p=0.657)$. Additionally, we explored specific effects for high-caloric vs. low-caloric as well as sweet vs. savory foods, but there were no group or stimulation effects specific for subgroups that would have survived correction for multiple comparisons across these exploratory analyses. The only effect that was significant at an uncorrected level was a Group $X$ Caloric Density interaction in wanting ratings, indicating stronger wanting of high-caloric vs. low-caloric food in patients with $\operatorname{MDD}(b=9.25, t(61)=2.19, p=0.033)$.

a

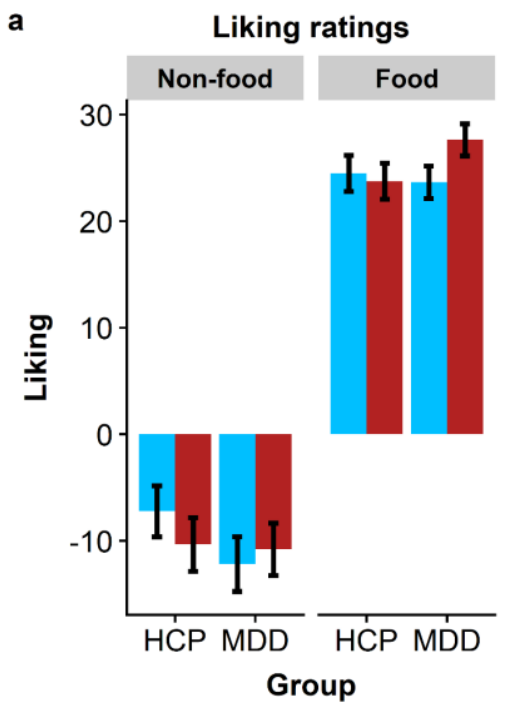

C

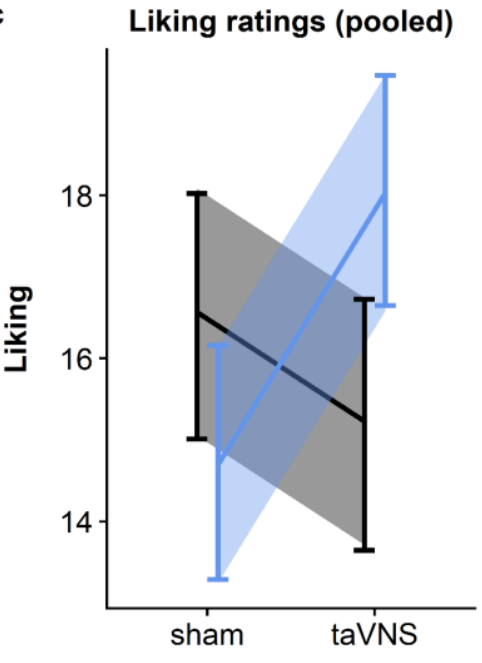

b
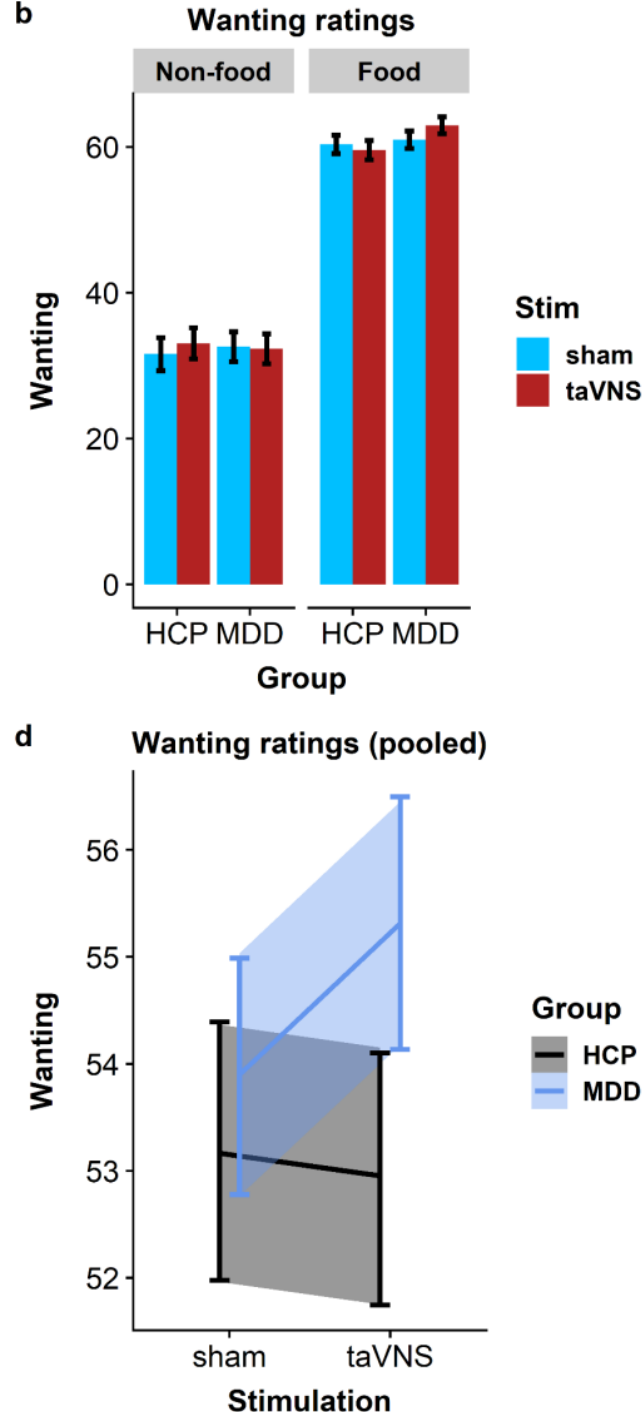

Figure 2. taVNS boosts food liking in participants with major depressive disorder (MDD) A: Food images were liked more compared to non-food images B: Food images were wanted more compared to nonfood images. C: taVNS increased food liking in the MDD $(t(60)=2.33, p=0.023)$, but not in the HCP group $(t(60)=-0.45, p=0.657$ ) (Group X Stimulation (pooled). $b=4.63, t(60)=2.43, p=0.018)$. D: taVNSinduced changes in wanting were similar, but less pronounced and non-significant (Group X Stimulation (pooled). $b=1.66, t(60)=0.86, p=0.396$ ). Depicted are means and $95 \%$ confidence intervals. 
taVNS increases food liking in participants with greater anhedonia and normalizes food liking across groups

Next, we examined associations of SHAPS and BAS scores on taVNS-induced changes in food liking and wanting. We entered SHAPS and BAS scores as participant-level predictors and included their cross-level interaction with stimulation (see Supplementary Information). During sham, SHAPS scores were not associated with food liking or wanting (liking: $b=-0.45, t(56)=-0.61, p=0.547$; wanting: $b=0.40, t(57)=0.69, p=0.495$ ). However, higher SHAPS scores were associated with higher taVNS-induced increases in food liking (SHAPS $\times$ Stimulation. $b=1.01, t(59)=2.23, p=0.029$ ), but not wanting (SHAPS $\times$ Stimulation. $b=0.20, t(59)=0.45, p=0.656)$. As expected, during sham, higher BAS scores were associated with higher food wanting $(b=0.69, t(57)=2.34, p=0.023)$, but not significantly with liking ratings $(b=0.69, t(57)=1.87, p=0.067$; Fig. $4 \mathrm{c}-\mathrm{d})$. However, in contrast to anhedonia, taVNS-induced changes in food liking (BAS X Stimulation. $b=-0.31, t(59)=-1.39, p=0.169$ ) and wanting (BAS $\times$ Stimulation. $b=-0.29, t(59)=-1.34, p=0.187)$ were not significantly associated with BAS scores.

Analogous to the significant interaction between anhedonia and stimulation effects, individual stimulation effects might depend on the individual's average food reward ratings. In support of a broader role in normalizing food reward ratings, we observed a significant taVNSinduced reduction in variance of liking $\left(\chi^{2}(1)=4.25, p=0.039\right.$, Fig. 5), but not wanting $\left(\chi^{2}(1)=\right.$ $0.03, p=0.871$ ) across participants. This reduction in variance (i.e., the convergence of liking ratings after taVNS) was driven by a negative correlation between liking rating intercepts (i.e., average ratings) and individual taVNS slopes (i.e., changes induced by the stimulation, $r=-$ 0.35). Thus, taVNS seems to boost lower and attenuate higher liking ratings (Fig. 5a,c). 

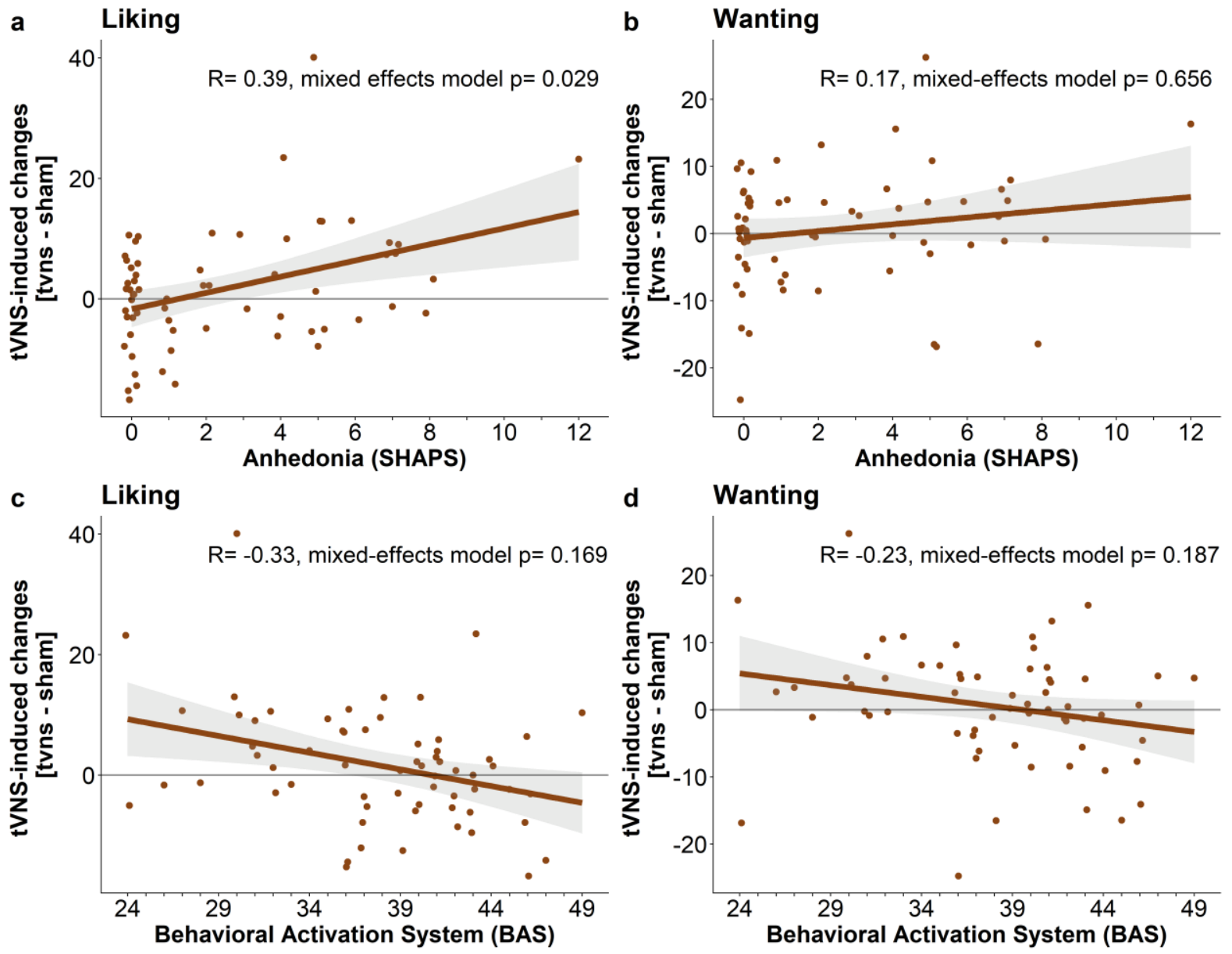

Figure 4. taVNS effects on liking ratings depend on anhedonia (SHAPS), but not behavioral activation (BAS). The plots show the difference between individual mean ratings of food images during taVNS vs. sham as a function of questionnaire scores. Positive values represent an increase of ratings following taVNS. A: taVNS-induced changes in liking increase with higher levels of anhedonia $(b=1.01, t(59)=$ 2.23, $p=0.029)$. B: taVNS-induced changes in wanting did not depend on anhedonia levels $(b=0.20$, $t(59)=0.45, p=0.656)$. C: taVNS effects on liking ratings did not depend on BAS scores $(b=-0.31, t(59)=$ $-1.39, p=0.169)$. D: taVNS effects on wanting ratings were also not associated with BAS scores $(b=-$ $0.29, t(59)=-1.34, p=0.187)$. 
a

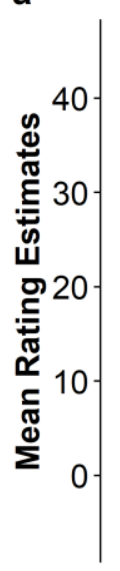

Liking

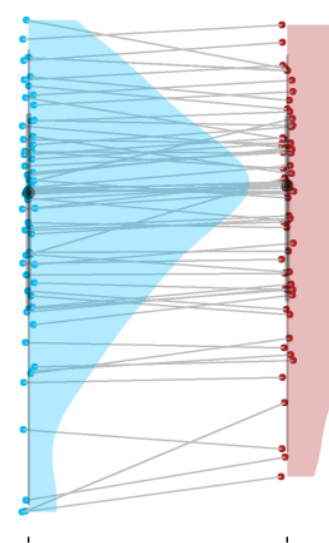

Intercept

taV'NS

C

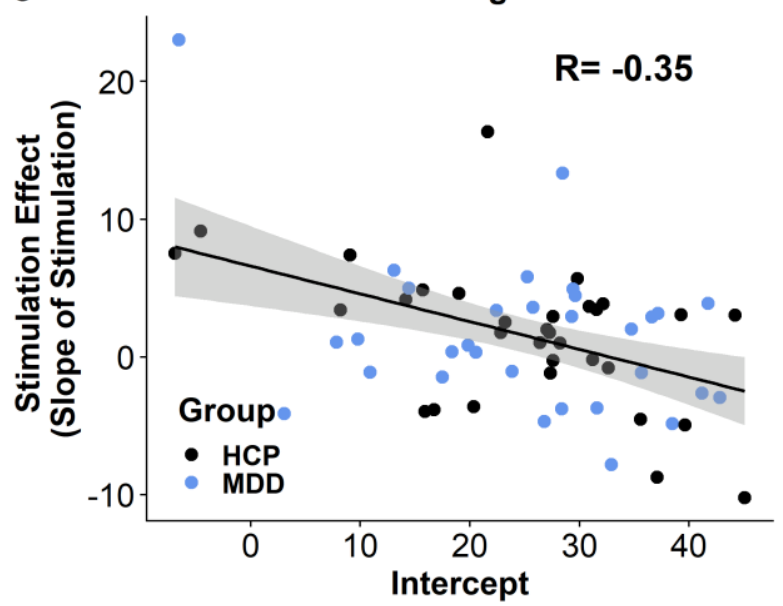

b

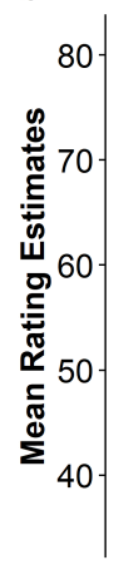

d
Wanting

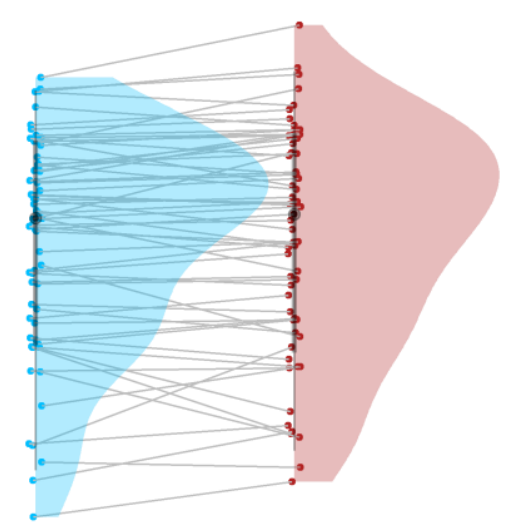

taV'NS

Intercept

Wanting

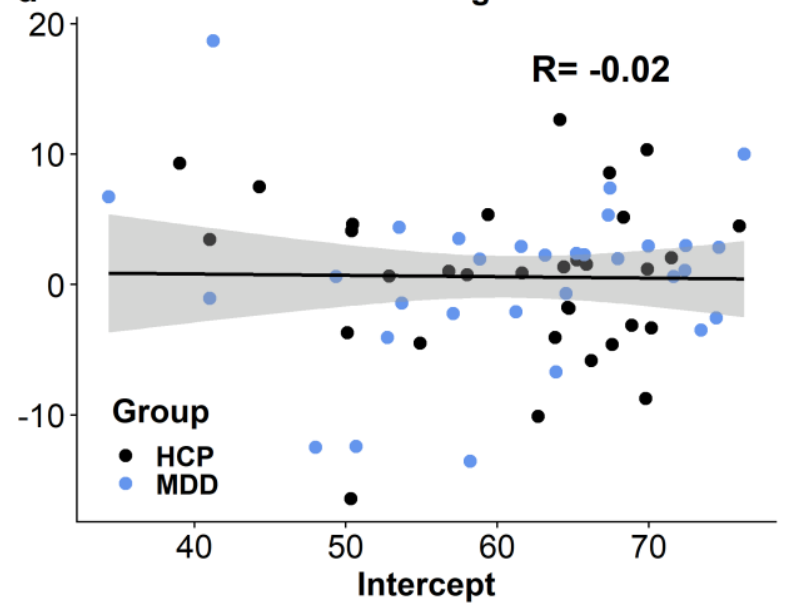

Figure 5. taVNS normalizes liking ratings. A: taVNS leads to a significant reduction in variance of liking ratings $\left(\chi^{2}(1)=4.25, p=0.039\right)$. B: No taVNS-induced changes in the variance of wanting ratings $\left(\chi^{2}(1)=\right.$ $0.03, p=0.871)$. C: Lower liking ratings at the intercept are associated with more positive and higher intercept liking ratings with more negative stimulation effects, driving the convergence effect. D: Wanting ratings at the intercept are not associated with stimulation effects.

\section{Discussion}

Anhedonia is associated with altered hedonic responses to rewards and is hard to treat via common psychological and pharmacological interventions (Craske et al, 2016). Here, we investigated effects of acute taVNS on liking and wanting ratings of various food and non-food images in participants with and without MDD. In line with previous reports of antidepressive effects of VNS, we found that acute taVNS increased liking of food cues in participants suffering from MDD, but not in HCP. Crucially, greater anhedonia was associated with a stronger taVNS-induced increase in food liking. Intriguingly, such a normalization was also evident across all participants since taVNS boosted low and attenuated high food liking. Taken 
together, our results indicate that taVNS acutely improves anhedonia in MDD, where most conventional treatments only achieve small and delayed effectiveness (Craske et al., 2016; Uher et al., 2012).

Our result that non-invasive VNS increases food liking specifically in patients with MDD and, in general, participants with low average food liking and higher scores of anhedonia helps reconcile conflicting earlier findings in human research. On the one hand, altered ratings of sweet food in patients with MDD have been reported after invasive VNS (Bodenlos et al., 2007), indicating that VNS may both increase and decrease food liking, conceivably depending on individual characteristics. On the other hand, we and others have reported no robust taVNS effects on average food ratings in healthy participants (Alicart et al., 2020; F.K. Müller et al., 2021; Obst et al., 2020). One potential explanation for these results may be the reported normalizing effect of taVNS on liking ratings, indicating that taVNS can either increase or decrease liking, depending on the average strength of hedonic responses, which varies considerably between people and even within MDD (Rizvi et al., 2016; Swiecicki et al., 2009; Treadway \& Zald, 2011). Crucially, taVNS-induced increases in liking were stronger with increasing levels of anhedonia, a core diagnostic symptom of MDD, that is less responsive to pharmacological treatment (Uher et al., 2012) and is a negative prognostic factor for treatment outcome (Spijker et al., 2001). Therefore, our results point to a potential therapeutic effect of taVNS on impairments in food hedonics, which may contribute to the antidepressive effects of taVNS (Wu et al., 2018).

The normalizing effect of taVNS on food liking raises the question which neurobiological mechanism may support differential effects of vagal afferent stimulation. While invasive VNS has mostly been associated with reduced food intake and weight reductions in animals and humans (Bugajski et al., 2007; Yao et al., 2018), recent preclinical work has shown that vagally induced activation of the NTS can either stimulate or suppress feeding depending on the neural population that is activated within the NTS (Chen et al., 2020). Thus, it is conceivable that the net effect of taVNS depends on the tuning of separable neural populations within the NTS, which might be affected by state-like (e.g., metabolic state) or trait-like differences (overall hedonic response to food; (Smith et al., 2017)). Additionally, we have recently shown that taVNS alters myoelectric activity (Teckentrup et al., 2020) in the stomach and increases stomach-brain coupling (S. Müller et al., 2021), suggesting that enhanced stomach-brain communication could prove as another candidate mechanism for the taVNS-induced normalization of aberrant hedonic responses to food. Notably, MDD is characterized by heterogeneous symptom profiles, including diverging changes in appetite and body weight during depressive episodes (Milaneschi et al., 2019; Simmons et al., 2016, 2020). Motivational deficiencies (including anhedonia) are more frequent in patients who suffer 
from loss of appetite and weight (Fletcher et al., 2015; Post \& Warden, 2018). Therefore, the normalization effect of taVNS might help recover vegetative symptoms in both melancholic (i.e., characterized by loss of appetite and weight) and atypical depression (i.e., characterized by an increase of appetite and weight). To summarize, taVNS could be a promising and versatile therapeutic tool to acutely normalize aberrant food hedonics and more research in humans is needed to better understand how differential taVNS effects could be maximized for future treatments.

Despite the notable strengths of our comparatively large taVNS cross-over study in patients with MDD and HCP, there are also several limitations of our study. First, we only investigated effects of taVNS on hedonic responses to food rewards and not on other rewards with comparable hedonic value. Since vagal signaling induces changes in dopamine transmission (de Araujo et al., 2012; Han et al., 2018) noradrenergic transmission (de Araujo et al., 2012; Han et al., 2018; Raedt et al., 2011; Roosevelt et al., 2006) and motivation (Neuser et al. 2020; Kühnel et al. 2020), taVNS might also affect hedonic and motivated responses beyond food, which remains to be tested in future research. Second, other state characteristics such as metabolic state may also affect acute taVNS effects on food ratings, which should be investigated systematically in the future to maximize treatment effects. Third, as we investigated the acute effects of taVNS on food reward, further research is needed to investigate a longer lasting impact of taVNS on anhedonia, preferably using longer periods of continuous stimulation.

In sum, we observed an increase in food liking specifically among participants with MDD and greater anhedonia. Likewise, we saw support for a normalizing effect of taVNS on food liking across the sample since higher liking ratings were attenuated and lower ratings were boosted during taVNS. We conclude that taVNS helps improve aberrant food reward responses while exerting little effect on moderate liking ratings, which may help reconcile conflicting previous findings in mostly healthy populations. Thereby, taVNS could be a promising therapeutic tool to acutely improve alterations in food reward processing. Our finding that greater anhedonia is associated with stronger taVNS-induced improvements might help allocate taVNS as an adjuvant treatment according to a patient's individual profile in the future, which is in line with calls for a more personalized approach in psychiatry (Carter et al., 2012; Green et al., 2017; Malhi \& John Mann, 2018). 


\section{Acknowledgement}

We thank Wy Ming Lin, Franziska Kräutlein, and Larissa Katz for help with data acquisition. The study was supported by the University of Tübingen, Faculty of Medicine fortune grant \#2453-0-0 and the Daimler and Benz Foundation postdoctoral scholarship 32-04/19 awarded to NBK. MF received salary support from the University of Tübingen, Faculty of Medicine IZKF Promotionskolleg.

\section{Author contributions}

NBK was responsible for the study concept and design. FKM, MF, \& JK collected data and recruited patients under supervision by NBK. NBK conceived the method and VK processed the data and performed the analysis while AK \& NBK contributed to analyses. VK, JK \& NBK wrote the manuscript. All authors contributed to the interpretation of findings, provided critical revision of the manuscript for important intellectual content, and approved the final version for publication.

\section{Financial disclosure}

The authors declare no competing financial interests. 


\section{References}

Alicart, H., Heldmann, M., Göttlich, M., Obst, M. A., Tittgemeyer, M., \& Münte, T. F. (2020). Modulation of visual processing of food by transcutaneous vagus nerve stimulation (tVNS). Brain Imaging and Behavior. https://doi.org/10.1007/s11682-020-00382-8

Beck, A. T., Steer, R. A., Brown, G. K., \& Others. (1996). Manual for the beck depression inventory-II. San Antonio, TX: Psychological Corporation.

Berthoud, H.-R. (2008). The vagus nerve, food intake and obesity. Regulatory Peptides, 149(1-3), 15-25.

Beck, A. T., Steer, R. A., Brown, G. K., \& Others. (1996). Manual for the beck depression inventory-II. San Antonio, TX: Psychological Corporation.

Bodenlos, J. S., Kose, S., Borckardt, J. J., Nahas, Z., Shaw, D., O’Neil, P. M., \& George, M. S. (2007). Vagus nerve stimulation acutely alters food craving in adults with depression. Appetite, 48(2), 145-153.

Bottomley, J. M., LeReun, C., Diamantopoulos, A., Mitchell, S., \& Gaynes, B. N. (2020). Vagus nerve stimulation (VNS) therapy in patients with treatment resistant depression: A systematic review and meta-analysis. Comprehensive Psychiatry, 98, 152156.

Breit, S., Kupferberg, A., Rogler, G., \& Hasler, G. (2018). Vagus Nerve as Modulator of the Brain-Gut Axis in Psychiatric and Inflammatory Disorders. Frontiers in Psychiatry / Frontiers Research Foundation, 9. https://doi.org/10.3389/fpsyt.2018.00044

Bugajski, A. J., Gil, K., Ziomber, A., Zurowski, D., Zaraska, W., \& Thor, P. J. (2007). Effect of long-term vagal stimulation on food intake and body weight during diet induced obesity in rats. Journal of Physiology and Pharmacology: An Official Journal of the Polish Physiological Society, 58 Suppl 1, 5-12.

Carter, G. C., Cantrell, R. A., Victoria Zarotsky, Haynes, V. S., Phillips, G., Alatorre, C. I., Goetz, I., Paczkowski, R., \& Marangell, L. B. (2012). Comprehensive review of factors implicated in the heterogeneity of response in depression. Depression and Anxiety, 29(4), 340-354.

Carver, C. S., \& White, T. L. (1994). Behavioral inhibition, behavioral activation, and affective responses to impending reward and punishment: The BIS/BAS Scales. Journal of Personality and Social Psychology, 67(2), 319-333.

Charbonnier, L., van Meer, F., van der Laan, L. N., Viergever, M. A., \& Smeets, P. A. M. (2016). Standardized food images: A photographing protocol and image database. Appetite, 96, 166-173.

Chaudhri, O. B., Salem, V., Murphy, K. G., \& Bloom, S. R. (2008). Gastrointestinal satiety signals. Annual Review of Physiology, 70, 239-255.

Chen, J., Cheng, M., Wang, L., Zhang, L., Xu, D., Cao, P., Wang, F., Herzog, H., Song, S., \& Zhan, C. (2020). A Vagal-NTS Neural Pathway that Stimulates Feeding. Current Biology: CB, 30(20), 3986-3998.e5.

Coccurello, R. (2019). Anhedonia in depression symptomatology: Appetite dysregulation and defective brain reward processing. Behavioural Brain Research, 372, 112041.

Craske, M. G., Meuret, A. E., Ritz, T., Treanor, M., \& Dour, H. J. (2016). Treatment for Anhedonia: A Neuroscience Driven Approach. Depression and Anxiety, 33(10), 927-938.

Crichton, N. (2001). Information point: Visual Analogue Scale (VAS). Journal of Clinical Nursing, 10, 697-706.

Dai, F., Yin, J., \& Chen, J. D. Z. (2020). Effects and Mechanisms of Vagal Nerve Stimulation on Body Weight in Diet-Induced Obese Rats. Obesity Surgery, 30(3), 948-956.

de Araujo, I. E., Ferreira, J. G., Tellez, L. A., Ren, X., \& Yeckel, C. W. (2012). The gut-brain dopamine axis: a regulatory system for caloric intake. Physiology \& Behavior, 106(3), 394-399.

de Lartigue, G., \& Diepenbroek, C. (2016). Novel developments in vagal afferent nutrient sensing and its role in energy homeostasis. Current Opinion in Pharmacology, 31, 38-43.

Ellrich, J. (2011). Transcutaneous Vagus Nerve Stimulation. European Neurological Review, 6(4), 254.

Farmer, A. D., Strzelczyk, A., Finisguerra, A., Gourine, A. V., Gharabaghi, A., Hasan, A., Burger, A. M., Jaramillo, A. M., Mertens, A., Majid, A., Verkuil, B., Badran, B. W., Ventura-Bort, C., Gaul, C., Beste, C., Warren, C. M., Quintana, D. S., Hämmerer, D., Freri, E., ... Koenig, J. (2020). International Consensus Based Review and Recommendations for Minimum Reporting Standards in Research on Transcutaneous Vagus Nerve Stimulation (Version 2020). Frontiers in Human Neuroscience, 14, 568051.

Ferstl, M., Teckentrup, V., Lin, W. M., Kräutlein, F., Kühnel, A., Klaus, J., Walter, M., \& Kroemer, N. B. (2021). Noninvasive vagus nerve stimulation boosts mood recovery after effort exertion. Psychological Medicine, 1-11.

Fletcher, K., Parker, G., Paterson, A., Fava, M., losifescu, D., \& Pizzagalli, D. A. (2015). Anhedonia in melancholic and non-melancholic depressive disorders. Journal of Affective Disorders, 184, 81-88. 
Frangos, E., Ellrich, J., \& Komisaruk, B. R. (2015). Non-invasive Access to the Vagus Nerve Central Projections via Electrical Stimulation of the External Ear: fMRI Evidence in Humans. Brain Stimulation, 8(3), 624-636.

Franz, M., Lemke, M. R., Meyer, T., Ulferts, J., Puhl, P., \& Snaith, R. P. (2008). Deutsche Version der SnaithHamilton-Pleasure-Scale (SHAPS-D). Fortschritte der Neurologie, Psychiatrie, und ihrer Grenzgebiete, 66(09), 407-413.

Fydrich, T., Wittchen, H.-U., \& Zaudig, M. (1997). SKID : Strukturiertes Klinisches Interview für DSM-IV; Achse I und II. SKID-II SKID-II. Hogrefe.

Green, E., Goldstein-Piekarski, A. N., Schatzberg, A. F., John Rush, A., Ma, J., \& Williams, L. (2017). Personalizing antidepressant choice by sex, body mass index, and symptom profile: An iSPOT-D report. In Personalized Medicine in Psychiatry (Vols. 1-2, pp. 65-73). https://doi.org/10.1016/j.pmip.2016.12.001

Han, W., Tellez, L. A., Perkins, M. H., Perez, I. O., Qu, T., Ferreira, J., Ferreira, T. L., Quinn, D., Liu, Z.-W., Gao, X.-B., Kaelberer, M. M., Bohórquez, D. V., Shammah-Lagnado, S. J., de Lartigue, G., \& de Araujo, I. E. (2018). A Neural Circuit for Gut-Induced Reward. Cell, 175(3), 665-678.e23.

Hasin, D. S., Sarvet, A. L., Meyers, J. L., Saha, T. D., Ruan, W. J., Stohl, M., \& Grant, B. F. (2018). Epidemiology of Adult DSM-5 Major Depressive Disorder and Its Specifiers in the United States. JAMA Psychiatry, 75(4), 336346.

Keedwell, P. A., Andrew, C., Williams, S. C. R., Brammer, M. J., \& Phillips, M. L. (2005). The Neural Correlates of Anhedonia in Major Depressive Disorder. In Biological Psychiatry (Vol. 58, Issue 11, pp. 843-853). https://doi.org/10.1016/j.biopsych.2005.05.019

Keller, J., Young, C. B., Kelley, E., Prater, K., Levitin, D. J., \& Menon, V. (2013). Trait anhedonia is associated with reduced reactivity and connectivity of mesolimbic and paralimbic reward pathways. Journal of Psychiatric Research, 47(10), 1319-1328.

Krohne, H. W., Egloff, B., Kohlmann, C.-W., \& Tausch, A. (1996). Untersuchungen mit einer deutschen Version der "Positive and Negative Affect Schedule" (PANAS). Diagnostica, 42, 139-156.

Kühnel, A., Teckentrup, V., Neuser, M. P., Huys, Q. J. M., Burrasch, C., Walter, M., \& Kroemer, N. B. (2020). Stimulation of the vagus nerve reduces learning in a go/no-go reinforcement learning task. European Neuropsychopharmacology: The Journal of the European College of Neuropsychopharmacology, 35, 17-29.

Kuznetsova, A., Brockhoff, P. B., \& Christensen, R. H. B. (2017). ImerTest Package: Tests in Linear Mixed Effects Models. Journal of Statistical Software, Articles, 82(13), 1-26.

Lenth, R. V. (2021). Estimated Marginal Means, aka Least-Squares Means [R package emmeans version 1.6.1]. https://CRAN.R-project.org/package=emmeans

Lim, J., Wood, A., \& Green, B. G. (2009). Derivation and evaluation of a labeled hedonic scale. Chemical Senses, 34(9), 739-751.

Malhi, G. S., \& John Mann, J. (2018). Depression. In The Lancet (Vol. 392, Issue 10161, pp. 2299-2312). https://doi.org/10.1016/s0140-6736(18)31948-2

Milaneschi, Y., Simmons, W. K., van Rossum, E. F. C., \& Penninx, B. W. (2019). Depression and obesity: evidence of shared biological mechanisms. Molecular Psychiatry, 24(1), 18-33.

Müller, F. K., Teckentrup, V., Kühnel, A., Ferstl, M., \& Kroemer, N. B. (2021). Acute vagus nerve stimulation does not affect liking or wanting ratings of food in healthy participants. In bioRxiv (p. 2021.03.26.437062). https://doi.org/10.1101/2021.03.26.437062

Müller, S., Teckentrup, V., Rebollo, I., Hallschmid, M., \& Kroemer, N. B. (2021). Vagus nerve stimulation increases stomach-brain coupling via a vagal afferent pathway. In bioRxiv (p. 2021.10.07.463517). https://doi.org/10.1101/2021.10.07.463517

Neuser, M. P., Teckentrup, V., Kühnel, A., Hallschmid, M., Walter, M., \& Kroemer, N. B. (2020). Vagus nerve stimulation boosts the drive to work for rewards. Nature Communications, 11(1), 3555.

Obst, M. A., Heldmann, M., Alicart, H., Tittgemeyer, M., \& Münte, T. F. (2020). Effect of Short-Term Transcutaneous Vagus Nerve Stimulation (tVNS) on Brain Processing of Food Cues: An Electrophysiological Study. Frontiers in Human Neuroscience, 14, 206.

Öztürk, L., Büning, P. E., Frangos, E., de Lartigue, G., \& Veldhuizen, M. G. (2020). tVNS Increases Liking of Orally Sampled Low-Fat Foods: A Pilot Study. Frontiers in Human Neuroscience, 14, 600995.

Pelot, N. A., \& Grill, W. M. (2018). Effects of vagal neuromodulation on feeding behavior. Brain Research, 1693(Pt B), 180-187.

Peters, J. H., Simasko, S. M., \& Ritter, R. C. (2006). Modulation of vagal afferent excitation and reduction of food intake by leptin and cholecystokinin. Physiology \& Behavior, 89(4), 477-485.

Peuker, E. T., \& Filler, T. J. (2002). The nerve supply of the human auricle. Clinical Anatomy, 15(1), 35-37. 
Pizzagalli, D. A. (2014). Depression, Stress, and Anhedonia: Toward a Synthesis and Integrated Model. In Annual Review of Clinical Psychology (Vol. 10, Issue 1, pp. 393-423). https://doi.org/10.1146/annurev-clinpsy-050212185606

Post, R. J., \& Warden, M. R. (2018). Melancholy, anhedonia, apathy: the search for separable behaviors and neural circuits in depression. In Current Opinion in Neurobiology (Vol. 49, pp. 192-200). https://doi.org/10.1016/j.conb.2018.02.018

Raedt, R., Clinckers, R., Mollet, L., Vonck, K., El Tahry, R., Wyckhuys, T., De Herdt, V., Carrette, E., Wadman, W., Michotte, Y., Smolders, I., Boon, P., \& Meurs, A. (2011). Increased hippocampal noradrenaline is a biomarker for efficacy of vagus nerve stimulation in a limbic seizure model. In Journal of Neurochemistry (Vol. 117, Issue 3, pp. 461-469). https://doi.org/10.1111/j.1471-4159.2011.07214.x

Rizvi, S. J., Pizzagalli, D. A., Sproule, B. A., \& Kennedy, S. H. (2016). Assessing anhedonia in depression: Potentials and pitfalls. Neuroscience and Biobehavioral Reviews, 65, 21-35.

R Core Team (2020). R: A language and environment for statistical computing. R Foundation for Statistical Computing, Vienna, Austria. URL https://www.R-project.org/.

Rømer Thomsen, K., Whybrow, P. C., \& Kringelbach, M. L. (2015). Reconceptualizing anhedonia: novel perspectives on balancing the pleasure networks in the human brain. Frontiers in Behavioral Neuroscience, 9 , 49.

Roosevelt, R. W., Smith, D. C., Clough, R. W., Jensen, R. A., \& Browning, R. A. (2006). Increased extracellular concentrations of norepinephrine in cortex and hippocampus following vagus nerve stimulation in the rat. Brain Research, 1119(1), 124-132.

Shankman, S. A., Katz, A. C., DeLizza, A. A., Sarapas, C., Gorka, S. M., \& Campbell, M. L. (2014). The Different Facets of Anhedonia and Their Associations with Different Psychopathologies. In Anhedonia: A Comprehensive Handbook Volume I (pp. 3-22). https://doi.org/10.1007/978-94-017-8591-4_1

Simmons, W. K., Burrows, K., Avery, J. A., Kerr, K. L., Bodurka, J., Savage, C. R., \& Drevets, W. C. (2016). Depression-Related Increases and Decreases in Appetite: Dissociable Patterns of Aberrant Activity in Reward and Interoceptive Neurocircuitry. The American Journal of Psychiatry, 173(4), 418-428.

Simmons, W. K., Burrows, K., Avery, J. A., Kerr, K. L., Taylor, A., Bodurka, J., Potter, W., Teague, T. K., \& Drevets, W. C. (2020). Appetite changes reveal depression subgroups with distinct endocrine, metabolic, and immune states. Molecular Psychiatry, 25(7), 1457-1468.

Smith, R., Thayer, J. F., Khalsa, S. S., \& Lane, R. D. (2017). The hierarchical basis of neurovisceral integration. Neuroscience and Biobehavioral Reviews, 75, 274-296.

Snaith, R. P., Hamilton, M., Morley, S., Humayan, A., Hargreaves, D., \& Trigwell, P. (1995). A scale for the assessment of hedonic tone the Snaith-Hamilton Pleasure Scale. The British Journal of Psychiatry: The Journal of Mental Science, 167(1), 99-103.

Sobocki, J., Królczyk, G., Herman, R. M., Matyja, A., \& Thor, P. J. (2005). Influence of vagal nerve stimulation on food intake and body weight--results of experimental studies. Journal of Physiology and Pharmacology: An Official Journal of the Polish Physiological Society, 56 Suppl 6, 27-33.

Spijker, J., Bijl, R. V., de Graaf, R., \& Nolen, W. A. (2001). Determinants of poor 1-year outcome of DSM-III-R major depression in the general population: results of the Netherlands Mental Health Survey and Incidence Study (NEMESIS). Acta Psychiatrica Scandinavica, 103(2), 122-130.

Strobel, A., Beauducel, A., Debener, S., \& Brocke, B. (2001). Eine deutschsprachige Version des BIS/BASFragebogens von Carver und White. Zeitschrift Für Differentielle Und Diagnostische Psychologie, 22(3), 216227.

Swiecicki, L., Zatorski, P., Bzinkowska, D., Sienkiewicz-Jarosz, H., Szyndler, J., \& Scinska, A. (2009). Gustatory and olfactory function in patients with unipolar and bipolar depression. Progress in Neuro-Psychopharmacology \& Biological Psychiatry, 33(5), 827-834.

Teckentrup, V., Neubert, S., Santiago, J. C. P., Hallschmid, M., Walter, M., \& Kroemer, N. B. (2020). Non-invasive stimulation of vagal afferents reduces gastric frequency. Brain Stimulation, 13(2), 470-473.

Treadway, M. T., \& Zald, D. H. (2011). Reconsidering anhedonia in depression: lessons from translational neuroscience. Neuroscience and Biobehavioral Reviews, 35(3), 537-555.

Uher, R., Perlis, R. H., Henigsberg, N., Zobel, A., Rietschel, M., Mors, O., Hauser, J., Dernovsek, M. Z., Souery, D., Bajs, M., Maier, W., Aitchison, K. J., Farmer, A., \& McGuffin, P. (2012). Depression symptom dimensions as predictors of antidepressant treatment outcome: replicable evidence for interest-activity symptoms. Psychological Medicine, 42(5), 967-980.

Val-Laillet, D., Biraben, A., Randuineau, G., \& Malbert, C. H. (2010). Chronic vagus nerve stimulation decreased 
weight gain, food consumption and sweet craving in adult obese minipigs. Appetite, 55(2), 245-252.

Vrieze, E., Demyttenaere, K., Bruffaerts, R., Hermans, D., Pizzagalli, D. A., Sienaert, P., Hompes, T., de Boer, P., Schmidt, M., \& Claes, S. (2014). Dimensions in major depressive disorder and their relevance for treatment outcome. Journal of Affective Disorders, 155, 35-41.

Waise, T. M. Z., Dranse, H. J., \& Lam, T. K. T. (2018). The metabolic role of vagal afferent innervation. Nature Reviews. Gastroenterology \& Hepatology, 15(10), 625-636.

Watson, D., Clark, L. A., \& Tellegen, A. (1988). Development and validation of brief measures of positive and negative affect: the PANAS scales. Journal of Personality and Social Psychology, 54(6), 1063-1070.

Westfall, Jake (https://stats.stackexchange.com/users/5829/jake-westfall), Mixed effects model: Compare random variance component across levels of a grouping variable, URL (version: 2019-05-13): https://stats.stackexchange.com/q/362878

Whiteford, H. A., Degenhardt, L., Rehm, J., Baxter, A. J., Ferrari, A. J., Erskine, H. E., Charlson, F. J., Norman, R. E., Flaxman, A. D., Johns, N., Burstein, R., Murray, C. J. L., \& Vos, T. (2013). Global burden of disease attributable to mental and substance use disorders: findings from the Global Burden of Disease Study 2010. The Lancet, 382(9904), 1575-1586.

Wickham, H. (2016). Programming with ggplot2. In H. Wickham (Ed.), ggplot2: Elegant Graphics for Data Analysis (pp. 241-253). Springer International Publishing.

Wu, C., Liu, P., Fu, H., Chen, W., Cui, S., Lu, L., \& Tang, C. (2018). Transcutaneous auricular vagus nerve stimulation in treating major depressive disorder. Medicine, 97(52). https://doi.org/10.1097/MD.0000000000013845

Yao, G., Kang, L., Li, J., Long, Y., Wei, H., Ferreira, C. A., Jeffery, J. J., Lin, Y., Cai, W., \& Wang, X. (2018). Effective weight control via an implanted self-powered vagus nerve stimulation device. Nature Communications, 9(1), 5349.

Young, C. B., Chen, T., Nusslock, R., Keller, J., Schatzberg, A. F., \& Menon, V. (2016). Anhedonia and general distress show dissociable ventromedial prefrontal cortex connectivity in major depressive disorder. Translational Psychiatry, 6, e810.

Yuan, H., \& Silberstein, S. D. (2016). Vagus Nerve and Vagus Nerve Stimulation, a Comprehensive Review: Part I. Headache, 56(1), 71-78. 